\title{
Alisol B 23-acetate inhibits the viability and induces apoptosis of non-small cell lung cancer cells via PI3K/AKT/mTOR signal pathway
}

\author{
YANG LIU $^{1}$, XI-CHAO XIA ${ }^{1}$, LIU-YANG MENG ${ }^{2}$, YU WANG $^{1}$ and YUE-MEI LI ${ }^{1}$ \\ ${ }^{1}$ Department of Medicine, Pingdingshan University; ${ }^{2}$ Emergency Department, \\ Pingdingshan Second People's Hospital, Pingdingshan, Henan 467000, P.R. China
}

Received September 29, 2018; Accepted February 14, 2019

DOI: $10.3892 / \mathrm{mmr} .2019 .10355$

\begin{abstract}
The aim of the present study was to investigate the effects of alisol B 23-acetate (AB23A) on inhibiting the viability and inducing apoptosis of human non-small cell lung cancer (NSCLC) cells and the anticancer mechanisms of AB23A in vitro. The viability of A549 cells following treatment with different doses of AB23A was examined using a Cell Counting Kit- 8 assay. Subsequently, apoptosis and the cell cycle were detected using flow cytometric analysis. The effect of AB23A on migration and invasion of A549 cells was detected by wound healing and Transwell assays. Western blotting was performed to determine the relative expression of $\mathrm{Bax} / \mathrm{Bcl}-2$, phosphatidylinositol 3-kinase (PI3K), protein kinase B (AKT) and mammalian target of rapamycin (mTOR). AB23A markedly inhibited the viability enhanced apoptosis of A549 cells and arrested the cell cycle in G1 phase. Additionally, AB23A upregulated the ratio of Bax/Bcl-2 in the A549 cells in a concentration-dependent manner. The results of wound healing and Transwell assays indicated that AB23A also suppresses the migration and invasion ability of A549 cells. Furthermore, AB23A reduced the protein levels of phosphorylated AKT, PI3K and mTOR. In conclusion, AB23A exerted anti-cancer activity via inhibiting cells viability, migration and invasion, and promoting apoptosis. Therefore, AB23A is a potential antitumor drug for the treatment of NSCLC.
\end{abstract}

\section{Introduction}

Non-small cell lung cancer (NSCLC), which accounts for $>85 \%$ of newly diagnosed cases of lung cancer, is the leading cause of morbidity and cancer-associated mortality worldwide (1).

Correspondence to: Dr Yang Liu, Department of Medicine, Pingdingshan University, 2 Chongwen Road, Xinhua, Pingdingshan, Henan 467000, P.R. China

E-mail: 11503688@163.com

Key words: non-small cell lung cancer, alisol B 23-acetate, cell viability, cell apoptosis, cell cycle
Although there are a large number of clinical treatment options, the effectiveness of treatment for patients with NSCLC is unfavorable, with a 5-year relative survival rate of $18 \%$ (1). Chemotherapy, adjuvant radiotherapy and biological targeted therapy following surgical excision have improved the survival rate of patients with cancer. Chemotherapy is the most effective and common treatment method. Although a significant number of patients initially respond favorably to chemotherapy, the majority of them exhibit severe side-effects and de novo or acquired resistance to chemotherapeutic drugs, and conventional chemotherapeutic drugs do not provide a marked survival advantage for patients $(2,3)$. Therefore, there is an urgent requirement to develop a novel drug that can effectively treat NSCLC with fewer side-effects.

Metastasis and proliferation of cancer cells are the main causes of deterioration of patients with NSCLC as cancer cells can survive beyond the normal life span of a cell, have increased proliferation and resistance to chemotherapy and facilitate metastatic activity (4). In addition, defective apoptosis is recognized as the major criterion that contributes to the initiation and progression of cancer. The key proteins in this process are BCL2 associated X (Bax) and B-cell lymphoma (Bcl-2). Consequently, induction of apoptosis and inhibition of cell viability are promising strategies for treatment of cancer. The process is associated with various signaling pathways, including that of phosphatidylinositol 3-kinase (PI3K)/protein kinase B (AKT)/mammalian target of rapamycin (mTOR). A previous study reported that the $\mathrm{PI} 3 \mathrm{~K} / \mathrm{Akt} / \mathrm{mTOR}$ pathway is involved with different cellular processes, from cell growth or survival, to cell necrosis or apoptosis (5). Notably, natural products are considered a promising source for the development of novel anticancer drugs due to their potential effectiveness and low toxicity (6).

Chinese herbal medicine has gradually become an important modern clinical therapeutic approach for human diseases due to the strong pharmacological properties, which contribute to cancer chemotherapy (7). Alisol B 23-acetate (AB23A), a triterpenoid compound, exists naturally in the rhizomes of Alisma orientalis (8) and has been identified to have anti-cancer biological functions (9). Furthermore, AB23A had been demonstrated to possess anti-proliferative activity (10) and induced Bax gene nuclear translocation and 
apoptotic in PC-3 cells (4). In addition, a number of studies have demonstrated that AB23A has anti-hepatitis virus (11) and anti-bacterial (12) pharmacological activity. In human renal proximal tubular cells, alisol B-induced autophagy mediates apoptosis and nephrotoxicity through the PI3K/AKT/mTOR signaling pathway (13). However, the anticancer mechanism of $\mathrm{AB} 23 \mathrm{~A}$ remains unclear.

In the present study, the effects of AB23A on A549 cells were systematically investigated, including those on cell viability, migration and invasion, the cell cycle, apoptosis and the activity of the PI3K/AKT/mTOR signaling pathways. The results demonstrated that $\mathrm{AB} 23 \mathrm{~A}$ may be a promising compound for the treatment of NSCLC. To the best of our knowledge, this study is the first to demonstrate that AB23A exerts anticancer effects on NSCLC and to investigate the possible corresponding molecular mechanism.

\section{Materials and methods}

Materials. AB23A (High pressure liquid chromatography $\geq 98 \%$ ) was purchased from Shanghai Moqi Biological Technology Co., Ltd. (Shanghai, China). The Cell Counting Kit-8 (CCK-8; cat. no. C0039) was purchased from Beyotime Institute of Biotechnology (Haimen, China). The propidium iodide (PI)/RNase staining kit and the Annexin V-FITC/7AAD kit were all purchased from BD Biosciences (San Jose, CA, USA); All primary antibodies, including Bax (cat. no. ab53154; 1:1,000), Bcl-2 (cat. no. ab196495; 1:1,000), AKT (cat. no. ab38449; 1:1,000), phosphorylated (p)-AKT (cat. no. ab18206; 1:500), PI3K (cat. no. ab86714; 1:1,000), p-PI3K (cat. no. ab125633; 1:1,000), mTOR (cat. no. ab63552; 1:500), p-mTOR (cat. no. ab1093; 1:1,000) and GAPDH (cat. no. ab9484; 1:5,000), and horseradish peroxidase-conjugated anti-mouse IgG (cat. no. ab205719; 1:10,000) or anti-rabbit IgG (cat. no. ab205718; 1:5,000) secondary antibodies were purchased from Abcam (Cambridge, UK).

Cell culture. The human NSCLC cell line A549 and normal human lung epithelial cell line BEAS-2B were obtained from the American Type Culture Collection (Manassas, VA, USA). BEAS-2B cells were cultured in bronchial epithelial cell growth medium (Lonza Group, Ltd., Basel, Switzerland). A549 cells were cultured in Dulbecco's Modified Eagle's medium (DMEM; Gibco; Thermo Fisher Scientific, Inc., Waltham, MA, USA) with $10 \%$ fetal bovine serum (FBS; Gibco; Thermo Fisher Scientific, Inc.) and $1 \%$ penicillin-streptomycin in a standard incubator supplied with $5 \% \mathrm{CO}_{2}$ at $37^{\circ} \mathrm{C}$.

AB23A treatment experiment. AB23A were dissolved in dimethyl sulfoxide (DMSO). The A549 cells and BEAS-2B cells were seeded in 12 -well plates at a density of $6 \times 10^{5}$ cells/well. AB23A at concentrations of 6 and $9 \mathrm{mM}$ or the vehicle (vehicle control, $1 \%$ DMSO) was added to the culture medium. The cells were then harvested for each experiment.

Cell growth rate assay. A CCK- 8 assay was conducted to measure cell viability and proliferation. Briefly, A549 cells ( $2 \times 10^{4}$ cells/well) and BEAS-2B cells $\left(5 \times 10^{3}\right.$ cells/well) in the exponential growth were placed in a 96 -well plate overnight. At a confluence of $70-80 \%$, the A549 and BEAS-2B cells were incubated with different concentrations of AB23B $(0,6$ and $9 \mathrm{mM})$ for different times $(12,24$ and $48 \mathrm{~h})$. Following treatment, the CCK-8 reagent was added to each well for $2 \mathrm{~h}$ at $37^{\circ} \mathrm{C}$ according to the manufacturer's protocol. The growth rate of the A549 and BEAS-2B cells was determined by measuring the absorbance at $450 \mathrm{~nm}$ among the three group under a microplate reader (Bio-Rad Laboratories, Inc., Hercules, CA, USA) and was calculated as follows: Growth rate $(\%)=($ mean experimental absorbance/mean control absorbance) x100.

Cell apoptosis assay. Quantification of apoptotic cells was conducted using flow cytometry. Briefly, A549 cells were pretreated with $\mathrm{AB} 23 \mathrm{~A}$ for $24 \mathrm{~h}$ and then were harvested and stained with an Annexin-V-FITC/7-AAD kit (BD Biosciences) according to the manufacturer's protocol. The data acquisition and analysis were performed with CellQuest 5.0 software (BD Biosciences).

Cell cycle analysis. The cell cycle distributions of the A549 cells was determined using flow cytometry. A total of $1 \times 10^{6}$ cells were cultured in combination with various concentration of AB23A for $24 \mathrm{~h}$. Subsequently, the cells were harvested and washed with ice-cold PBS, then fixed in $70 \%$ ethanol for $2 \mathrm{~h}$ at $-20^{\circ} \mathrm{C}$. The cells were then washed again with PBS and incubated with the PI/RNase solution for $25 \mathrm{~min}$ at $37^{\circ} \mathrm{C}$. The cell cycle distributions were detected using a FACSCAN laser flow cytometer equipped with CellQuest software version 5.0 (BD Biosciences).

Wound healing assay. The migration ability of the A549 cells was evaluated using a wound healing assay. When $70-80 \%$ of the 6-well plate was covered, the cells were vertically scraped with a $200 \mu$ l pipette tip. Subsequently, the cells were treated with various concentrations of AB23A. Images of three randomly selected fields along the scraped line in each well were captured using a camera (Nikon Corporation, Tokyo, Japan). Following incubation for 24 and $48 \mathrm{~h}$, further images of the selected fields were captured. The relative width of the wound in the 6 and $9 \mathrm{mM}$ groups compared with the vehicle control $(0 \mathrm{mM})$ group at 0,24 and $48 \mathrm{~h}$ was determined using ImageJ version $1.51 \mathrm{j} 8$ software (National Institutes of Health, Bethesda, MD, USA).

Transwell assay. The invasion activity of the A549 cells were assessed using a 24-well Transwell assay. An insert that was precoated with $100 \mu 1$ Matrigel and dried for $30 \mathrm{~min}$ at $37^{\circ} \mathrm{C}$ was placed in the upper chamber. Subsequently, A549 cells ( $2 \times 10^{4}$ cells/well) were suspended in DMEM with $0.5 \%$ FBS and various concentrations of $\mathrm{AB} 23 \mathrm{~A}$, and deposited into the upper chamber of each well; DMEM with $10 \%$ FBS was added to the lower chambers. After 24 and $48 \mathrm{~h}$ incubation, the cells remaining on the upper surface of the membranes were scraped off and the invasive cells on the lower surface of the membranes were fixed in $4 \%$ paraformaldehyde at $4{ }^{\circ} \mathrm{C}$ and stained with $0.1 \%$ crystal violet (Beyotime Institute of Biotechnology) for $25 \mathrm{~min}$ at room temperature. Images of three randomly selected fields were captured with a camera (Nikon Corporation). The invasion activity of the A549 cells was assessed by counting the number of cells under a light microscope (magnification, $\mathrm{x} 200$ ). 
Protein isolation and western blot analysis. Following treatment with various concentrations of AB23A (0, 6 and $9 \mathrm{mM})$ for 24 and $48 \mathrm{~h}$, the A549 cells were harvested, washed with ice-cold PBS and lysed in radioimmunoprecipitation assay lysis buffer (Thermo Fisher Scientific, Inc.) for $30 \mathrm{~min}$. The cell lysates were separated with centrifugation at $3,000 \mathrm{x} \mathrm{g}$ at $4^{\circ} \mathrm{C}$ for $15 \mathrm{~min}$ and then the supernatants were collected. The protein concentration was determined by a bicinchoninic acid (BCA) protein assay (Thermo Fisher Scientific, Inc.). Equivalent amounts of samples containing $40 \mu \mathrm{g}$ protein from each lysate were resolved by $10 \%$ SDS-PAGE. Subsequently, the protein was transferred onto a polyvinylidene difluoride membrane (Thermo Fisher Scientific, Inc.). After blocking with 5\% non-fat milk in PBS containing $0.1 \%$ Tween-20 (TPBS) at room temperature for $2 \mathrm{~h}$, the membranes were incubated overnight at $4^{\circ} \mathrm{C}$ with specific primary antibodies. The membranes were then washed with TPBS and incubated with the corresponding secondary antibodies for $1 \mathrm{~h}$ at room temperature. After washing the membranes again with TPBS, the protein bands were detected by an enhanced chemiluminescence method using a Tanon 4200R automatic chemiluminescence image analysis system (Tanon Science and Technology Co., Ltd., Shanghai, China) and ImageJ version $1.51 \mathrm{j} 8$ software was used to quantify protein expression.

Statistical analysis. Statistical analyses were performed with SPSS 19.0 software (IBM, Corps., Armonk, NY, USA). Data were presented as the mean \pm standard deviation of at least three independent experiments. Statistical differences were determined by a Student's t-test or one-way analysis of variance with Dunnett's multiple comparison post hoc test. $\mathrm{P}<0.05$ was considered to indicate a statistically significant difference.

\section{Results}

Effects of AB23A on the growth rate of human NSCLC cells, A549 and normal human lung epithelial cells, BEAS-2B. To evaluate the cytotoxicity of AB23A on A549 and BEAS-2B cells, a CCK-8 assay was used to measure cell growth. As presented in Fig. 1A, the growth rate of the A549 cells was significantly reduced by the various concentrations of AB23A (6 and $9 \mathrm{mM})$ in a time-dependent manner $(\mathrm{P}<0.01)$. Therefore, AB23A was cytotoxic to the A549 cells. The results also revealed that $\mathrm{AB} 23 \mathrm{~A}$ inhibited the growth rate of the A549 cells in a dose-dependent manner. The growth rate of the A549 cells was reduced to $50 \%$ following treatment with $9 \mathrm{mM}$ AB23A for $24 \mathrm{~h}$ (Fig. 1B). As presented in Fig. 1C, the growth rate of the BEAS-2B cells exhibited no significant change following treatment with the various concentrations of AB23A (6 and $9 \mathrm{mM}$ ) in a time-dependent manner. However, AB23A also demonstrated some cytotoxicity on the BEAS-2B cells, which lead to a slight decrease in its activity, although there was no statistically significance difference.

AB23A induces arrest of A549 cell cycle progression. AB23A induces an inhibitory effect through specific disturbances of cell cycle-associated events. To determine the effect of AB23A treatment on the progression of A549 cells cycle, flow cytometry was performed. As presented in Fig. 2, treatment with AB23A (6 and $9 \mathrm{mM}$ ) for $24 \mathrm{~h}$ led to a significant increase in the proportion of cells at the $\mathrm{G} 0 / \mathrm{G} 1$ phase $(\mathrm{P}<0.01)$ and
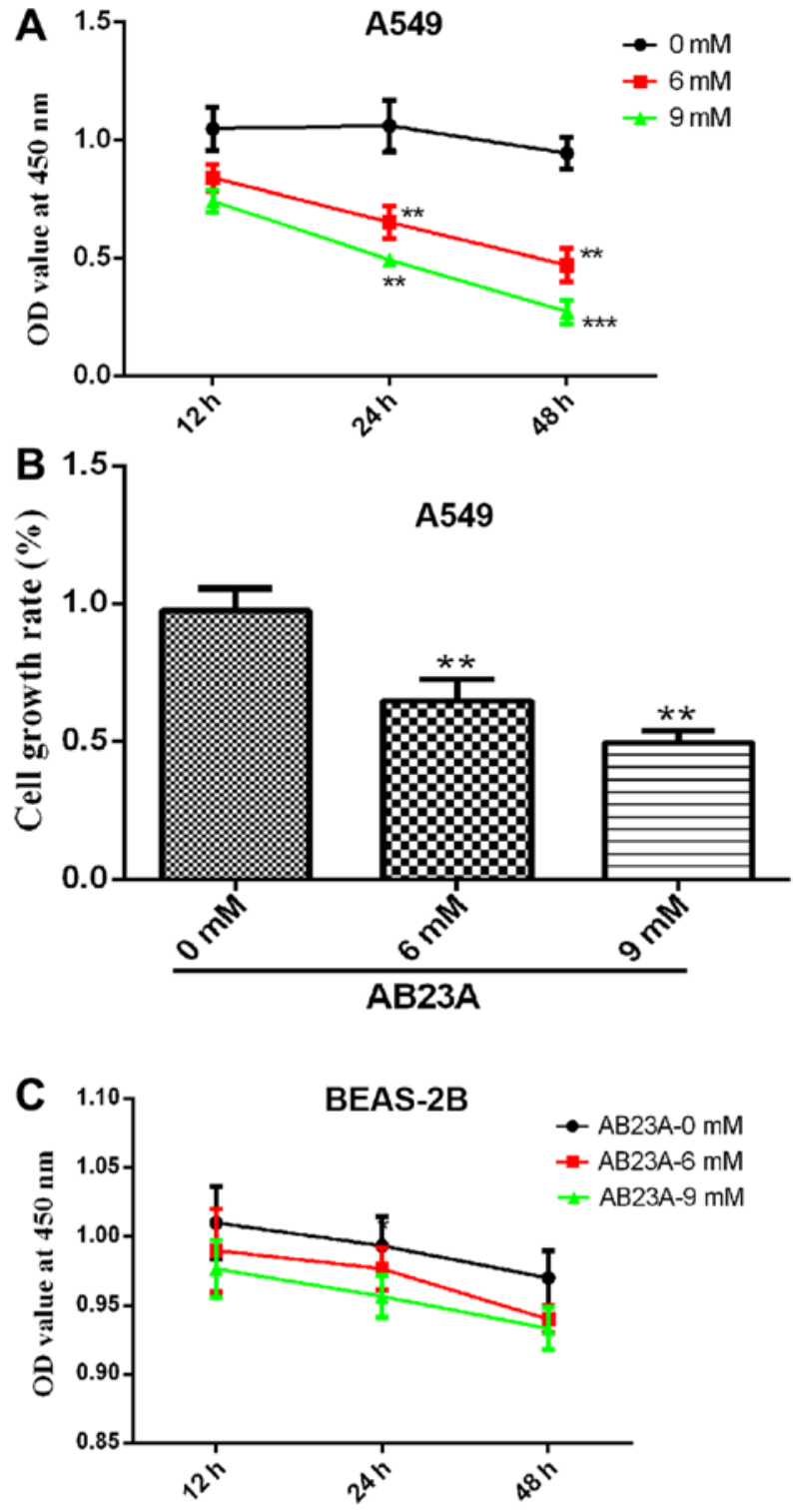

Figure 1. Effects of AB23A on the growth rate of A549 and BEAS-2B cells. (A) The growth rate of A549 cells following exposure to AB23A (0,6 and $9 \mathrm{mM}$ ) for 12,24 and $48 \mathrm{~h}$. (B) Histograms summarizing the results following treatment with $9 \mathrm{mM} \mathrm{AB} 23 \mathrm{~A}$ at $24 \mathrm{~h}$ in A. (C) The growth rate of BEAS-2B cells following exposure to AB23A $(0,6$ and $9 \mathrm{mM})$ for 12,24 and $48 \mathrm{~h}$. Data represent the mean \pm standard deviation of at least three independent experiments. ${ }^{* *} \mathrm{P}<0.01$ and ${ }^{* * *} \mathrm{P}<0.001$ vs. the vehicle control group $(0 \mathrm{mM})$. OD, optical density; AB23A, alisol B 23-acetate.

significantly reduced arrest in the $\mathrm{S}$ phase in a dose-dependent manner $(\mathrm{P}<0.05)$.

AB23A induces A549 cell apoptosis. After the cells were treated with different concentrations of AB23A (6 and $9 \mathrm{mM})$ for $24 \mathrm{~h}$, the percentage of apoptotic cells was detected using flow cytometry. Treatment with $9 \mathrm{mM}$ AB23A induced a significant increase in the percent of apoptotic cells $(\mathrm{P}<0.001$; Fig. 3). To clarify this effect, the expression levels of the apoptosis-associated proteins $\mathrm{Bax}$ and $\mathrm{Bcl}-2$ were detected using western blotting analysis. The levels of Bcl-2 expression were markedly downregulated and those of Bax expression were significantly elevated following treatment with various concentrations of AB23A (6 and $9 \mathrm{mM})$ for $24 \mathrm{~h}(\mathrm{P}<0.05$; 

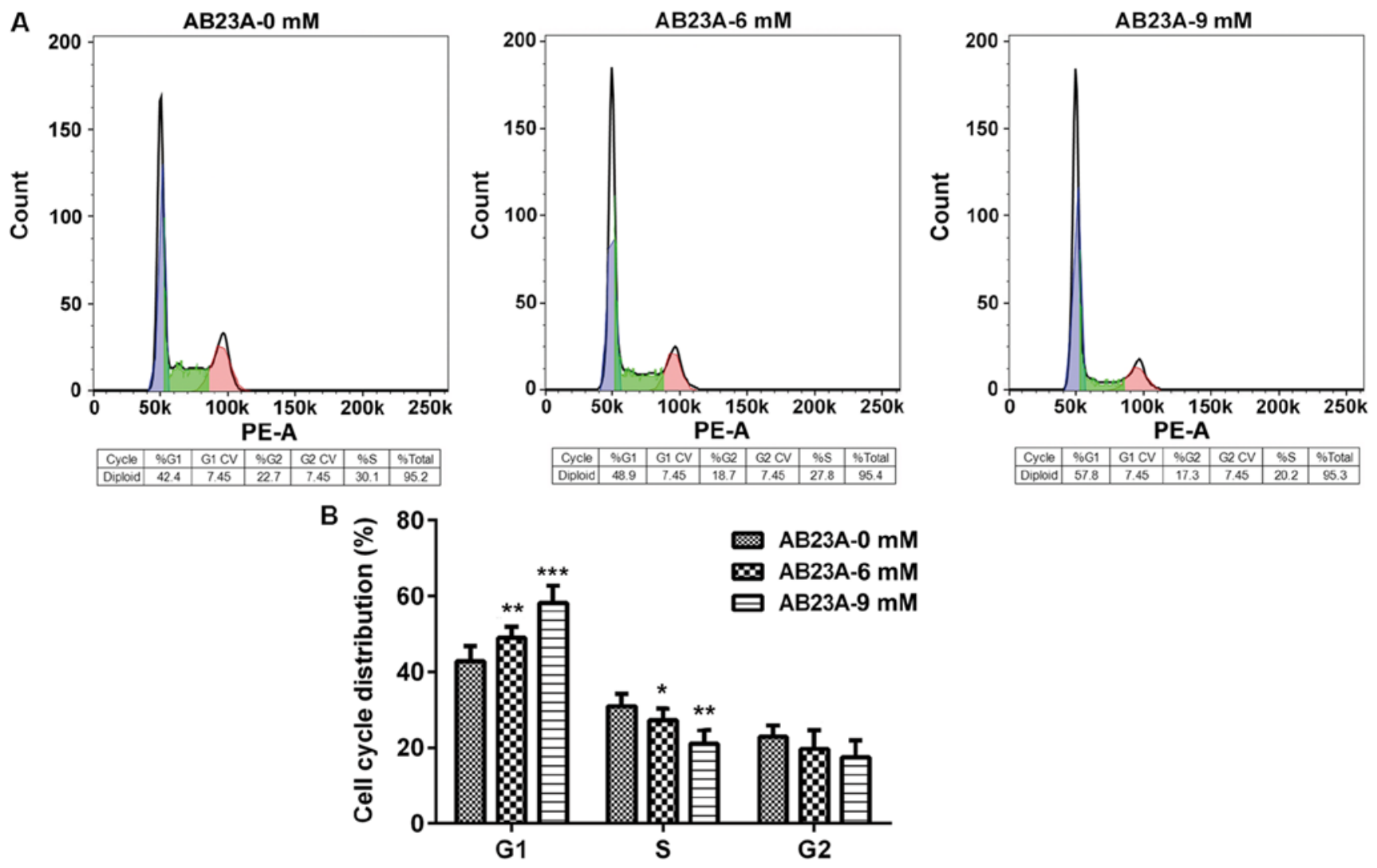

Figure 2. Effects of AB23A on cell cycle in A549 cells. A549 cells were permeabilized and stained with propidium iodide for cell cycle analysis by flow cytometry. (A) Representative picture of cell cycle analysis. (B) Histograms summarizing the results presented in A. The percentage of cells in G0/G1, S and G2/M was determined from the histogram displaying the cell number. Data are presented as the mean \pm standard deviation of three independent experiments. ${ }^{*} \mathrm{P}<0.05,{ }^{* * *} \mathrm{P}<0.01$ and ${ }^{* * * *} \mathrm{P}<0.001$ vs. the vehicle control group $(0 \mathrm{mM})$. AB23A, alisol B 23 -acetate.
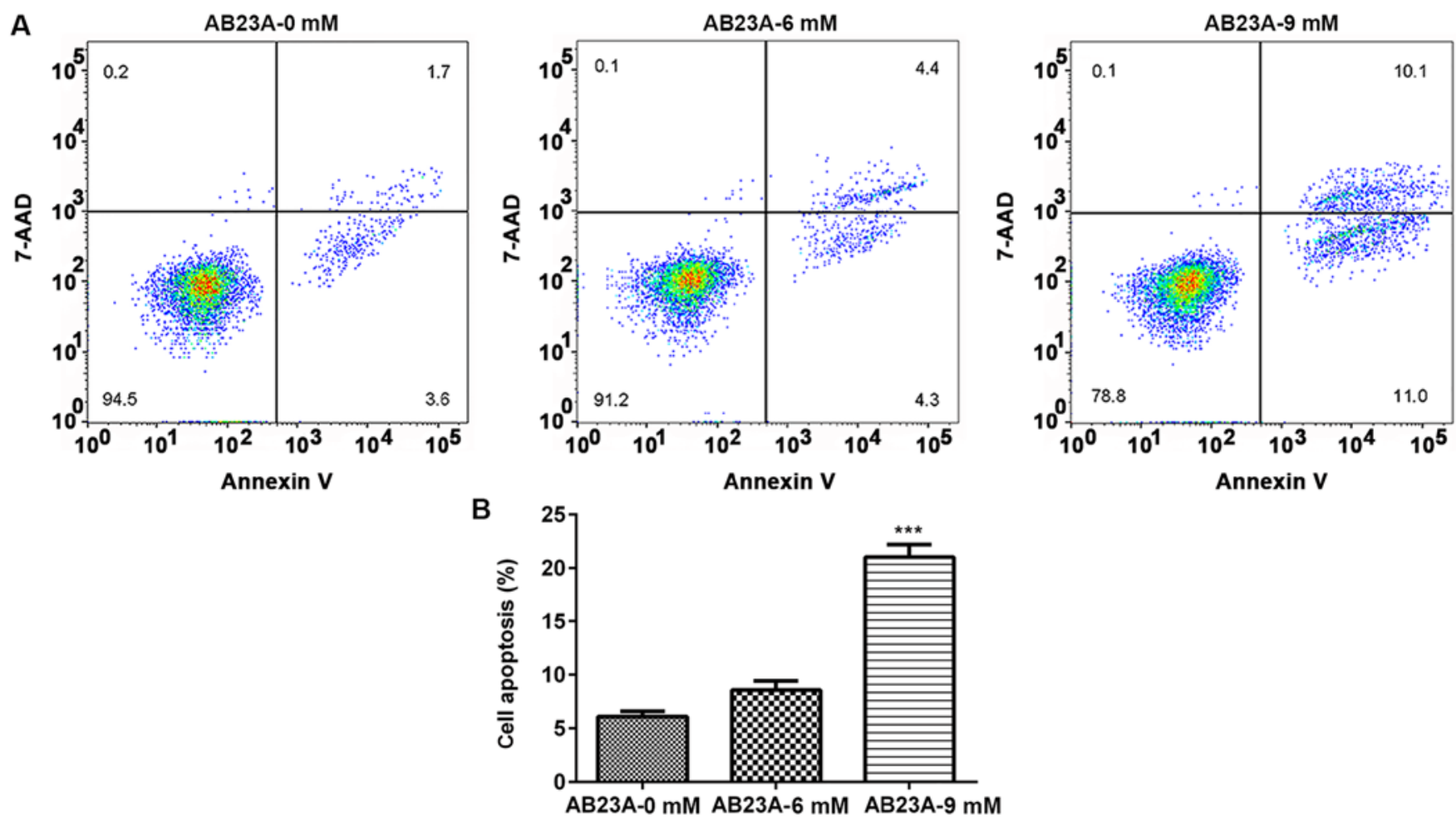

Figure 3. Effects of AB23A on apoptosis in A549 cells. (A) A549 cells were cultured in the presence of AB23A (0, 6 and 9 mM) for 24 h. Then, cells were harvested, washed with ice-cold PBS and stained using the Annexin-V-fluorescein isothiocyanate/7AAD kit. (B) Quantified histograms display the effect of AB23A on A549 apoptosis. Data are presented as the mean \pm standard deviation of three independent experiments. ${ }^{* * *} \mathrm{P}<0.001$ vs. the vehicle control group $(0 \mathrm{mM})$. AB23A, alisol B 23-acetate. 
A

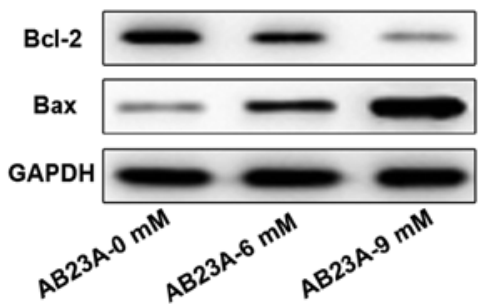

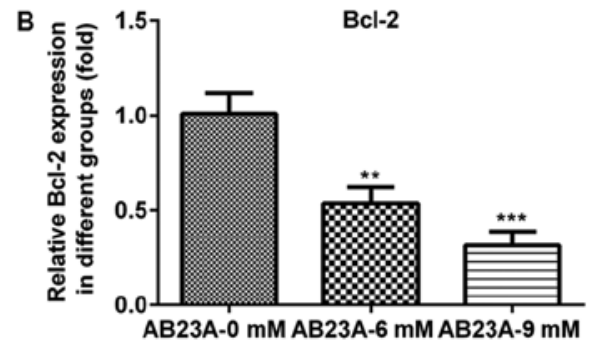

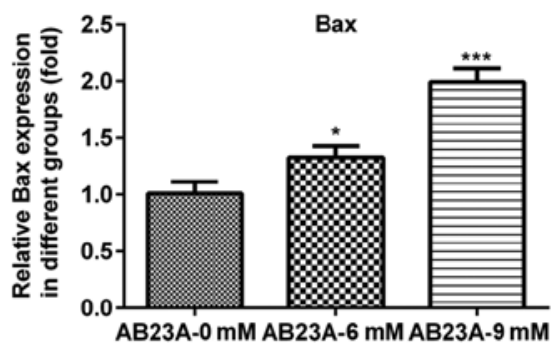

Figure 4. Effects of AB23A on the protein levels of Bax and Bcl-2 in A549 cells. (A) Representative pictures of western blots demonstrating the levels of Bax and Bcl-2. (B) Histograms summarizing the results presented in A. Data are presented as the mean \pm standard deviation of three independent experiments ${ }^{*} \mathrm{P}<0.05,{ }^{* *} \mathrm{P}<0.01$ and ${ }^{* * *} \mathrm{P}<0.001$ vs. the vehicle control group $(0 \mathrm{mM})$. Bcl-2, B-cell lymphoma; Bax, BCL2 associated X; AB23A, alisol B 23-acetate; AB23A, alisol B 23-acetate.

A
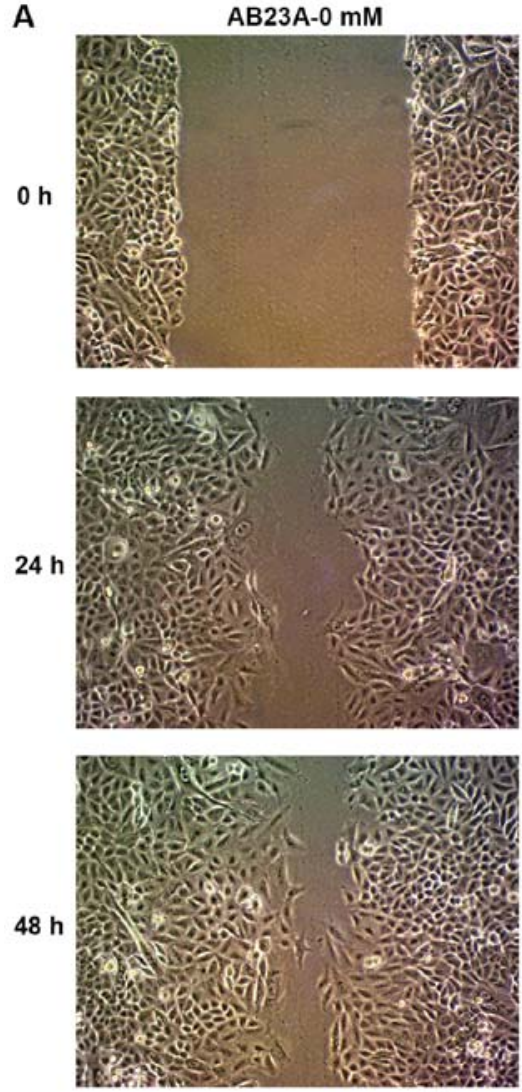
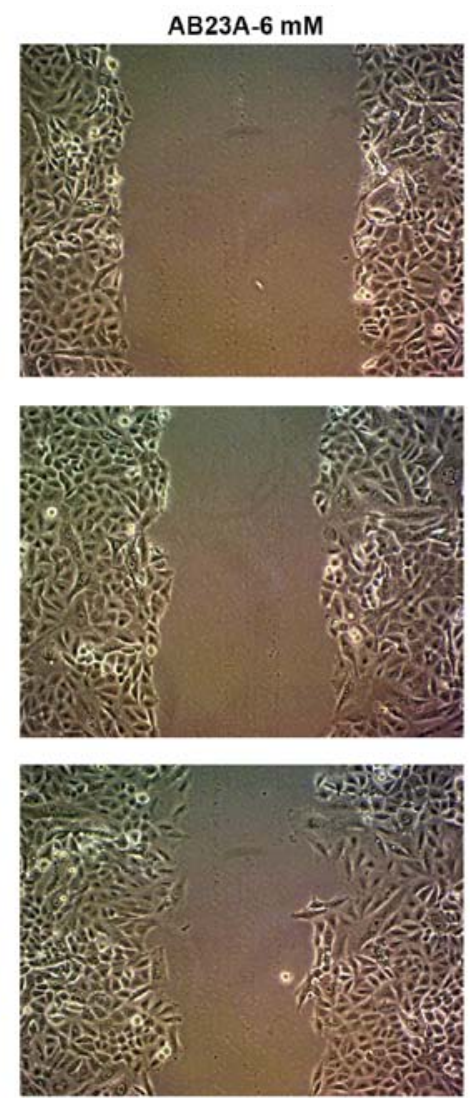

B

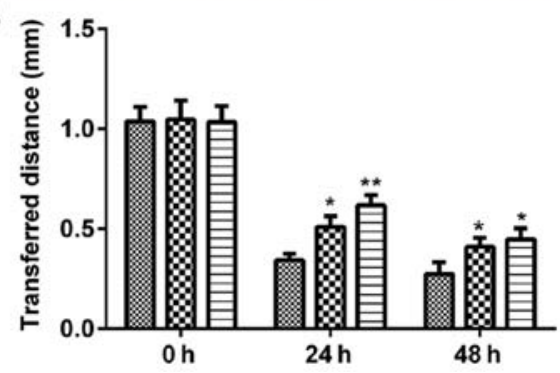

Figure 5. Effects of AB23A on migration in A549 cells. (A) Representative pictures of wound healing demonstrated that AB23A inhibited significantly cell migration following exposure to AB23A (0,6 and $9 \mathrm{mM}$ ) for 24 and $48 \mathrm{~h}$ (magnification, $\mathrm{x} 200)$. (B) Histograms summarizing the results presented in A. Data are presented as the mean \pm standard deviation of three independent experiments. ${ }^{*} \mathrm{P}<0.05$ and ${ }^{* *} \mathrm{P}<0.01$ vs. the vehicle control group $(0 \mathrm{mM})$. AB23A, alisol B 23 -acetate.

Fig. 4). These results suggested that AB23A induces apoptosis of NSCLC cells.

AB23A suppresses A549 cell migration and invasion. The migration and invasion abilities of A549 cells are important for NSCLC metastasis; therefore, the impact of AB23A on the abilities of the A549 cells was assessed using a wound healing assay and Transwell assay. The mobility and invasion of the A549 cells were significantly reduced following treatment with AB23A (6 and $9 \mathrm{mM})$ for 24 and $48 \mathrm{~h}(\mathrm{P}<0.05$; Figs. 5 and 6$)$. 

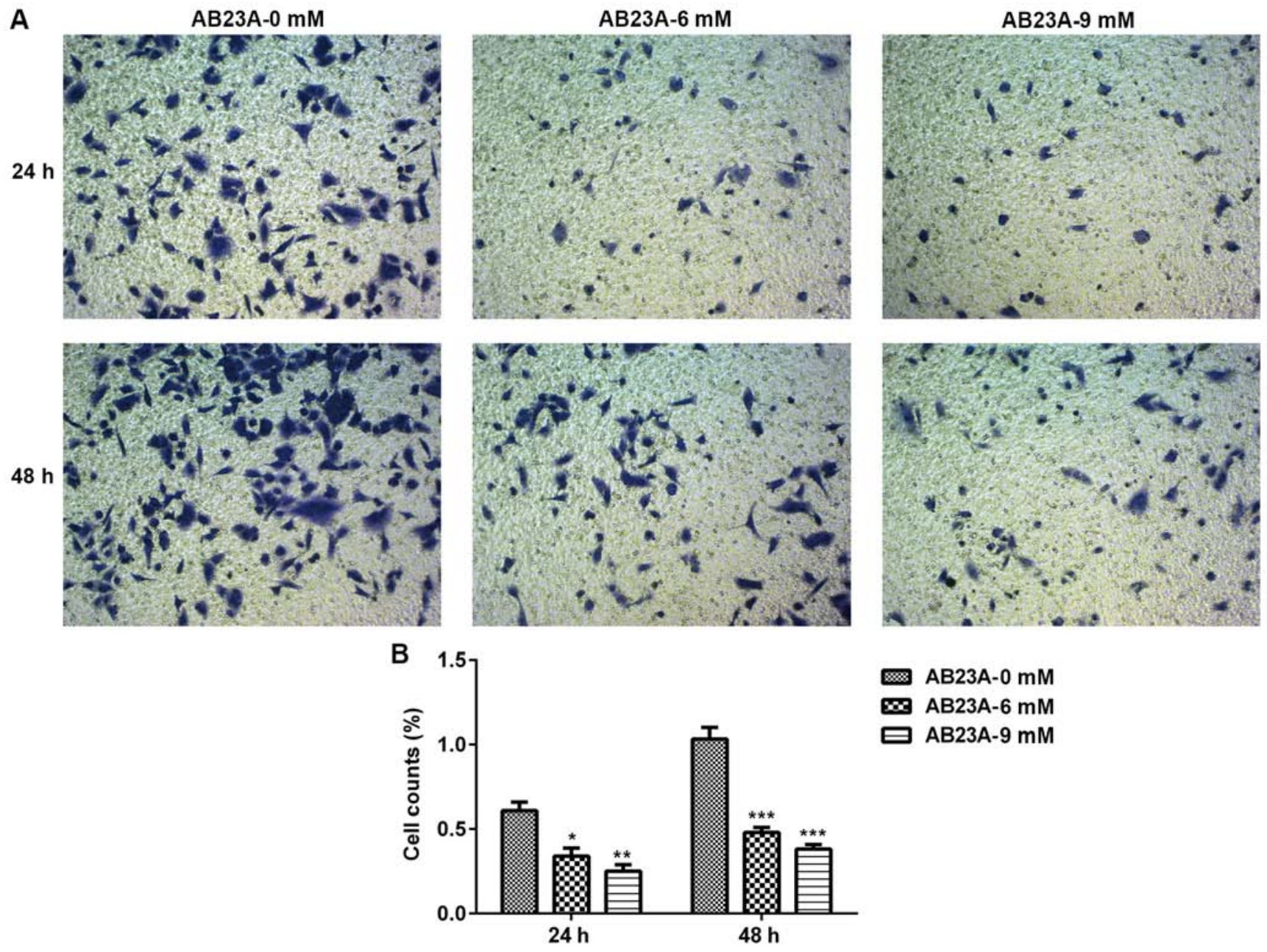

Figure 6. Effects of AB23A on invasion in A549 cells. (A) Representative pictures of Transwell assay demonstrated that AB23A inhibited significantly cells invasion following exposure to AB23A (0,6 and $9 \mathrm{mM}$ ) for 24 and $48 \mathrm{~h}$ (magnification, $\mathrm{x} 200)$. (B) Histograms summarizing the results presented in A. Data are presented as the mean \pm standard deviation of three independent experiments. ${ }^{*} \mathrm{P}<0.05,{ }^{* *} \mathrm{P}<0.01$ and ${ }^{* * * *} \mathrm{P}<0.001$ vs. the vehicle control group $(0$ mM). AB23A, alisol B 23-acetate.

AB23A reduces the phosphorylation levels of PI3K, AKT and mTOR in A549 cells. To investigate the mechanism of the apoptosis induced by AB23A, western blot analysis was performed to detect proteins in the $\mathrm{PI} 3 \mathrm{~K} / \mathrm{AKT} / \mathrm{mTOR}$ signaling pathways. As presented in Fig. 7, upon treatment with $\mathrm{AB} 23 \mathrm{~A}$ (6 and $9 \mathrm{mM}$ ) for 24 and $48 \mathrm{~h}$, the phosphorylation levels of PI3K, AKT and mTOR were remarkably reduced in a dose-and time-dependent manner, however, the levels of PI3K, AKT and mTOR were not significantly affected.

\section{Discussion}

The present study demonstrates, for the first time to the best of our knowledge, the therapeutic effects of AB23A on NSCLC cells. The majority of patients with NSCLC are treated with chemotherapy drugs, including platinum-based drugs; however, the side-effects of chemotherapy drugs reduced the survival rate of patients. Toxicity, drug resistance and a high risk of death have been observed in the clinic as side-effects of chemotherapy drugs used to treat NSCLC (14). Resistance of NSCLC to chemotherapy drugs has become a difficult issue to overcome. In such a severe situation, the natural products used in traditional Chinese medicine exhibit clear advantages, including few side-effects. Therefore, it is imperative to develop novel chemotherapy drugs to overcome refractory chemotherapy resistance and the side-effects of the current chemotherapeutic drugs.

Traditional Chinese medicine has the advantages of low cost, wide safety range, low toxicity, broad spectrum and numerous targets. Therefore, a number of researchers have investigated the rich resources of Chinese medicinal herbs (15). A previous study demonstrated that $\mathrm{AB} 23 \mathrm{~A}$ can inhibit nitric oxide production without cytotoxic effects (16). AB23A is commonly used for treatment of a number of diseases, including diabetes, pyelonephritis, inflammation and cancer $(17,18)$. The results of the present study revealed that the growth rate was reduced to $50 \%$ when the A549 cells were treated with $9 \mathrm{mM}$ AB23A for $24 \mathrm{~h}$, which indicates that AB23A has an inhibitory effect on cell vitality. In addition, in the present study, the inhibitory effect of AB23A on the activity of normal human lung epithelial cells (BEAS-2B) is not obvious, which indicates the low toxicity of AB23A to human body. However, it cannot be excluded that a high dose of AB23A will cause cytotoxicity to normal cells. Additionally, although the A549 and BEAS-2B growth rate reduced a little between 12 and $48 \mathrm{~h}$ in untreated cells (AB23A $0 \mathrm{mM}$ ), there was no significant difference and 
A

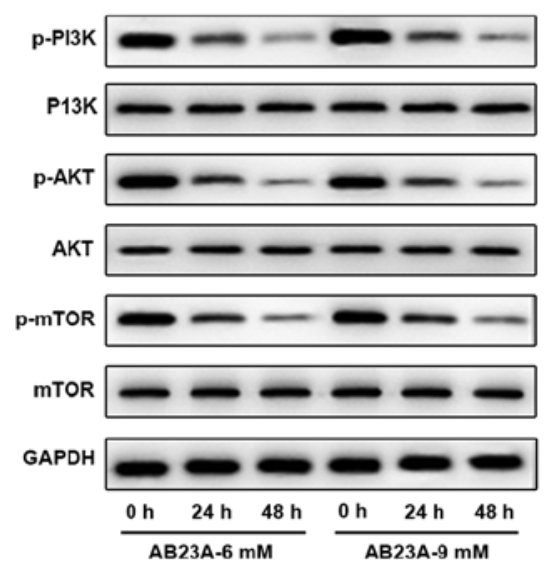

$\mathrm{B}$
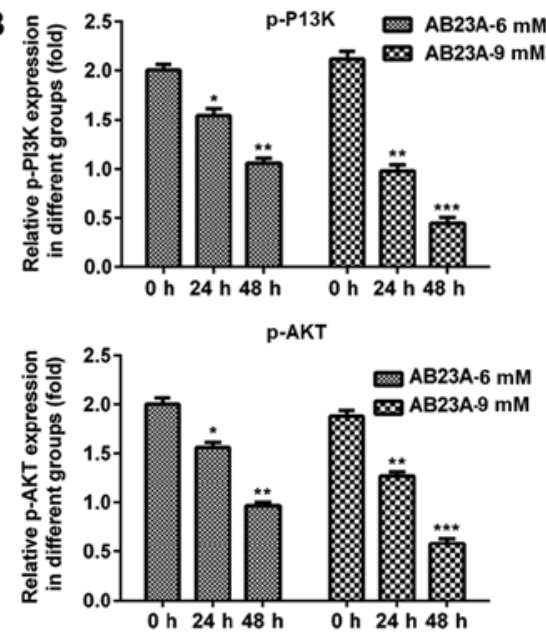

p-mTOR

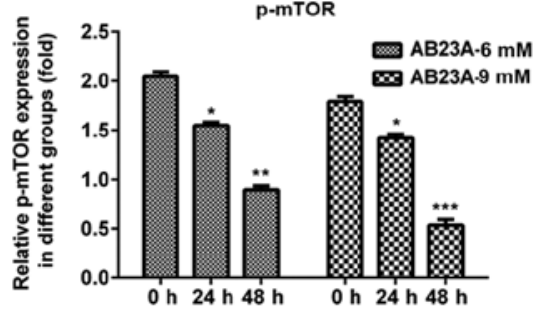

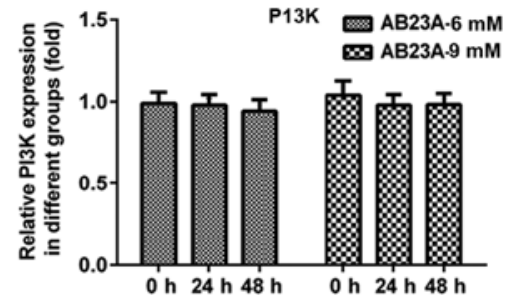

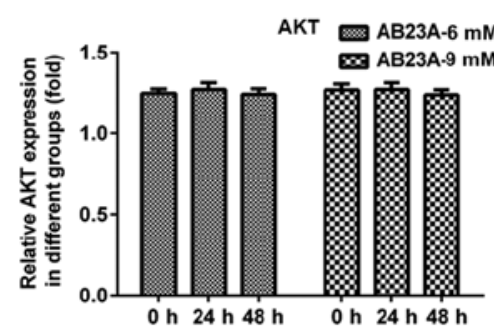

mTOR

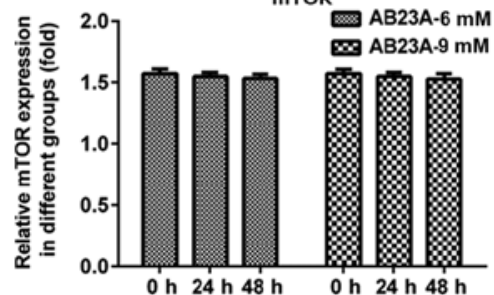

Figure 7. Effects of AB23A on PI3K/AKT/mTOR signaling pathways in A549 cells. (A) Representative pictures of western blots demonstrating the levels of total and phosphorylated of PI3K, AKT and mTOR. (B) Histograms summarizing the results presented in A. Data are presented as the mean \pm standard of three independent experiments. ${ }^{*} \mathrm{P}<0.05,{ }^{* *} \mathrm{P}<0.01$ and ${ }^{* * * *} \mathrm{P}<0.001$ vs. the vehicle control group $(0 \mathrm{~h})$. mTOR, mammalian target of rapamycin; AKT, protein kinase B; PI3K, phosphatidylinositol 3 kinase; p, phosphorylated; AB23A, alisol B 23-acetate.

the survival rate of the cells was all $>95 \%$. The present study hypothesized that the change in cell viability may be due to the slight effect of the DMSO solution on A549 cells. These all requires further research.

A previous study demonstrated that $\mathrm{AB} 23 \mathrm{~A}$ inhibits the proliferation of human breast cells through inducing apoptosis (19). AB23A also induces apoptosis, blocked the G1 phase, and inhibits migration and invasion of ovarian cancer cells (20). The apoptosis-associated genes Bax and Bcl-2 serve a vital role in the initiation and maintenance of apoptosis (21). Interference with cell cycle progression is one of the characteristics of numerous anticancer drugs. AB23A has been demonstrated to induce cell cycle arrest in the G1 phase in human colon cancer and ovarian cancer cell lines $(20,22)$. The results of the present study indicate that AB23A markedly induces cell cycle arrest in the G0/G1 phase in A549 cells. In addition, the results of the western blotting and flow cytometric analyses demonstrate that $\mathrm{AB} 23 \mathrm{~A}$ induces apoptosis and elevates the protein levels of $\mathrm{Bax} / \mathrm{Bcl}-2$ in a dose-dependence manner. Therefore, analysis of apoptosis and the cell cycle may be vital for developing AB23A as a potential anticarcinogen.

Activation of migration, invasion and metastasis is a crucial characteristic of malignancy, which is one of the hallmark capabilities of cancer (23). The high morbidity and mortality rates of patients with NSCLC are closely associated with the alterations observed in the biological behavior of lung cancer cells. Due to the high metastasis rate of cancer cells, NSCLC is difficult to eradicate. Therefore, inhibition of the biological behavior of NSCLC cells is a promising treatment for lung cancer. Wound healing and Transwell assays have been widely used to assess cancer cells metastasis and invasiveness in vitro $(24,25)$. In the present study, the results revealed that $\mathrm{AB} 23 \mathrm{~A}$ may markedly suppress the migration and invasion of A549 cells in a concentration-dependent manner following treatment for 24 and $48 \mathrm{~h}$. Additionally, cell apoptosis was evaluated following treatment for $24 \mathrm{~h}$, and apoptosis significantly increased in a concentration-dependent manner. When detecting the invasion and migration of A549 cells, it was observed that the cytotoxicity of AB23A on A549 cells was effected by inducing apoptosis and reducing cell growth, invasion and migration. Determining the underlying mechanisms requires further study.

The PI3K/AKT/mTOR pathway serves critical roles in diverse cellular processes, from proliferation, transcription, migration or survival to cell death or apoptosis, which are involved in cancer (26). Out of the molecules associated with this pathway, AKT is an important regulator of cellular activities that can be activated by PI3K and that phosphorylate a number of key pro-cancer factors to promote cell viability and inhibit apoptosis. mTOR belongs to the PI3K-related kinase family and is a vital kinase for regulating cell proliferation, apoptosis and viability (27). AKT is localized upstream of mTOR and the suppression of AKT phosphorylation can result in an significant reduction in phosphorylation of downstream mTOR (28). It is well known that mTOR serves an important role in regulating autophagy in mammalian signaling (29) and mTOR signaling is very important for cellular growth in response to a number of physiological conditions through regulating downstream effectors to control the translation and transcription of a variety of proteins (30). These key signaling molecules from this pathway serve a vital role in regulating cell survival and apoptosis. Drugs that target the PI3K/Akt/mTOR signaling pathway have the 
potential to inhibit survival pathways and induce apoptosis in cancer cells (16). Previous studies have reported that apigenin could inhibit the growth and proliferation, promote apoptotic cell death, induce cell cycle arrest via the PI3K/Akt/mTOR signaling pathway $(31,32)$. To further clarify the possible mechanism by which AB23A induces apoptosis of NSCLC cells, the impact of $\mathrm{AB} 23 \mathrm{~A}$ on the expression levels of PI3K, AKT and mTOR was investigated in the present study. Upregulation of Akt phosphorylation by the activation of PI3K and mTOR can integrate upstream activating signals through the PI3K/AKT pathway in return to result in phosphorylation (33). In the present study, AB23A induced apoptosis and inhibited the viability of the A549 cells via inhibition of the PI3K/AKT/mTOR signal pathway. Furthermore, alterations in the expression of phosphorylated $\mathrm{PI} 3 \mathrm{~K} / \mathrm{AKT} / \mathrm{mTOR}$ proteins and alterations in the cell phenotype in response to the AB23A stimulus were investigated. The results demonstrated that following treatment with AB23A (6 and $9 \mathrm{mM}$ ) for 24 and $48 \mathrm{~h}$, the protein levels of p-PI3K, p-AKT and p-mTOR were significantly reduced in the A549 cells, but there was no significant difference in the levels of PI3K, AKT and mTOR. In conclusion, treatment of NSCLC cells with AB23A has an anti-tumor effect, reduces cell viability and induces apoptosis via the activation of the PI3K/AKT/mTOR signaling pathway. The results reveal that $\mathrm{AB} 23 \mathrm{~A}$ could be an effective anticancer drug for the treatment of NSCLC.

In conclusion, the present study demonstrated that the anticancer activity of AB23A in A549 cells is mediated by the induction of apoptosis, interference with the cell cycle and suppression of cell activities, accompanied by inhibition of the $\mathrm{PI} 3 \mathrm{~K} / \mathrm{AKT} / \mathrm{mTOR}$ pathway in vitro. However, further studies are required to elucidate the underlying mechanism of action of $\mathrm{AB} 23 \mathrm{~A}$ and its anticancer activity in vivo also requires further evaluation. The results of this study may provide a novel therapeutic strategy for the treatment of human NSCLC.

\section{Acknowledgements}

We thank Vice President Zheng Xinhua of Pingdingshan College Medical College and Director Wang Hao of Pingdingshan Second People's Hospital for their guidance in the design of the scheme and the experimental operation.

\section{Funding}

No funding was received.

\section{Availability of data and materials}

All data generated or analyzed during the present study are included in this published article.

\section{Authors' contributions}

YL designed the experiments and drafted the manuscript. YL, XCX and LYM performed the experiments. YL, YW and YML analyzed the data.

\section{Ethics approval and consent to participate}

Not applicable.

\section{Patient consent for publication}

Not applicable.

\section{Competing interests}

The authors declare that they have no competing interests.

\section{References}

1. Siegel RL, Miller KD and Jemal A: Cancer statistics, 2017. CA Cancer J Clin 67: 7-30, 2017.

2. Chaudhary UB and Haldas JR: Long-term complications of chemotherapy for germ cell tumours. Drugs 63: 1565-1577, 2003.

3. Brouwers EE, Huitema AD, Beijnen JH and Schellens JH: Long-term platinum retention after treatment with cisplatin and oxaliplatin. BMC Clin Pharmacol 8: 7, 2008

4. Huang YT, Huang DM, Chueh SC, Teng CM and Guh JH: Alisol $\mathrm{B}$ acetate, a triterpene from Alismatis rhizoma, induces Bax nuclear translocation and apoptosis in human hormone-resistant prostate cancer PC-3 cells. Cancer Lett 231: 270-278, 2006.

5. Oral O, Akkoc Y, Bayraktar O and Gozuacik D: Physiological and pathological significance of the molecular cross-talk between autophagy and apoptosis. Histol Histopathol 31: 479-498, 2016.

6. Crowell JA: The chemopreventive agent development research program in the Division of Cancer Prevention of the US National Cancer Institute: An overview. Eur J Cancer 41: 1889-1910, 2005.

7. Mann J: Natural products in cancer chemotherapy: Past, present and future. Nat Rev Cancer 2: 143-148, 2002.

8. Lee AY, Park JY, Chun JM, Moon BC, Kang BK, Seo YB, Shin HK and Kim HK: Optimization of extraction condition for alisol B and alisol B acetate in alismatis rhizoma using response surface methodology. J Liq Chromatogr Relat Technol 36: 513-524, 2013.

9. Law BY, Wang M, Ma DL, Al-Mousa F, Michelangeli F, Cheng SH, Ng MH, To KF, Mok AY, Ko RY, et al: Alisol B, a novel inhibitor of the sarcoplasmic/endoplasmic reticulum $\mathrm{Ca}(2+)$ ATPase pump, induces autophagy, endoplasmic reticulum stress, and apoptosis. Mol Cancer Ther 9: 718-730, 2010.

10. Xu W, Li T, Qiu JF, Wu SS, Huang MQ, Lin LG, Zhang QW, Chen XP and Lu JJ: Anti-proliferative activities of terpenoids isolated from Alisma orientalis and their structure-activity relationships. Anticancer Agents Med Chem 15: 228-235, 2015.

11. Jiang ZY, Zhang XM, Zhang FX, Liu N, Zhao F, Zhou J and Chen JJ: A New triterpene and anti-hepatitis B virus active compounds from Alisma orientalis. Planta Med 72: 951-954, 2006.

12. Jin HG, Jin Q, Ryun Kim A, Choi H, Lee JH, Kim YS, Lee DG and Woo ER: A new triterpenoid from Alisma orientale and their antibacterial effect. Arch Pharm Res 35 : 1919-1926, 2012.

13. Wang C, Feng L, Ma L, Chen H, Tan X, Hou X, Song J, Cui L, Liu D, Chen J, et al: Alisol a 24-acetate and alisol B 23-acetate induced autophagy mediates apoptosis and nephrotoxicity in human renal proximal tubular cells. Front Pharmacol 8: 172, 2017.

14. Xiong Y, Huang BY and Yin JY: Pharmacogenomics of platinum-based chemotherapy in non-small cell lung cancer: Focusing on DNA repair systems. Med Oncol 34: 48, 2017.

15. Yang L, Yang C, Li C, Zhao Q, Liu L, Fang X and Chen XY: Recent advances in biosynthesis of bioactive compounds in traditional Chinese medicinal plants. Sci Bull (Beijing) 61: 3-17, 2016.

16. Xu W, Li X, Lin N, Zhang X, Huang X, Wu T, Tai Y, Chen S, Wu CH, Huang $\mathrm{M}$ and $\mathrm{Wu}$ S: Pharmacokinetics and tissue distribution of five major triterpenoids after oral administration of Rhizoma Alismatis extract to rats using ultra high-performance liquid chromatography-tandem mass spectrometry. J Pharm Biomed Anal 146: 314-323, 2017.

17. Zhang X, Li XY, Lin N, Zhao WL, Huang XQ, Chen Y, Huang MQ, Xu W and Wu SS: Diuretic activity of compatible triterpene components of alismatis rhizoma. Molecules 22: pii: E1459, 2017.

18. Matsuda H, Kageura T, Toguchida I, Murakami T, Kishi A and Yoshikawa M: Effects of sesquiterpenes and triterpenes from the rhizome of Alisma orientale on nitric oxide production in lipopolysaccharide-activated macrophages: Absolute stereostructures of alismaketones-B 23-acetate and -C 23-acetate. Bioorg Med Chem Lett 9: 3081-3086, 1999. 
19. Zhang A, Sheng Y and Zou M: Antiproliferative activity of Alisol $\mathrm{B}$ in MDA-MB-231 cells is mediated by apoptosis, dysregulation of mitochondrial functions, cell cycle arrest and generation of reactive oxygen species. Biomed Pharmacother 87: 110-1107, 2017.

20. Zhang LL, Xu YL, Tang ZH, Xu XH, Chen X, Li T, Ding CY, Huang MQ, Chen XP, Wang YT, et al: Effects of alisol B 23-acetate on ovarian cancer cells: G1 phase cell cycle arrest, apoptosis, migration and invasion inhibition. Phytomedicine 23: 800-809, 2016.

21. Melo-Lima S, Lopes MC and Mollinedo F: ERK1/2 acts as a switch between necrotic and apoptotic cell death in ether phospholipid edelfosine-treated glioblastoma cells. Pharmacol Res 95-96: 2-11, 2015.

22. Zhao Y, Li ETS and Wang M: Alisol B 23-acetate induces autophagic-dependent apoptosis in human colon cancer cells via ROS generation and JNK activation. Oncotarget 8: 70239-70249, 2017.

23. Hanahan D and Weinberg RA: The hallmarks of cancer. Cell 100: $57-70,2000$

24. Fujimura K, Choi S, Wyse M, Strnadel J, Wright $T$ and Klemke R: Eukaryotic translation initiation factor 5A (EIF5A) regulates pancreatic cancer metastasis by modulating RhoA and Rho-associated kinase (ROCK) protein expression levels. J Biol Chem 290: 29907-29919, 2015.

25. Gutschner T, Hämmerle M, Eissmann M, Hsu J, Kim Y, Hung G, Revenko A, Arun G, Stentrup M, Gross M, et al: The noncoding RNA MALAT1 is a critical regulator of the metastasis phenotype of lung cancer cells. Cancer Res 73: 1180-1189, 2013.

26. Morgan TM, Koreckij TD and Corey E: Targeted therapy for advanced prostate cancer: Inhibition of the PI3K/Akt/mTOR pathway. Curr Cancer Drug Targets 9: 237-249, 2009.
27. Martin KA and Blenis J: Coordinate regulation of translation by the PI 3-kinase and mTOR pathways. Adv Cancer Res 86: 1-39, 2002.

28. Surviladze Z, Sterk RT, DeHaro SA and Ozbun MA: Cellular entry of human papillomavirus type 16 involves activation of the phosphatidylinositol 3-kinase/Akt/mTOR pathway and inhibition of autophagy. J Virol 87: 2508-2517, 2013.

29. Foster KG and Fingar DC: Mammalian target of rapamycin (mTOR): Conducting the cellular signaling symphony. J Biol Chem 285: 14071-14077, 2010.

30. He C and Klionsky DJ: Regulation mechanisms and signaling pathways of autophagy. Annu Rev Genet 43: 67-93, 2009.

31. Yu W, Sun H, Zha W, Cui W, Xu L, Min Q and Wu J: Apigenin attenuates adriamycin-induced cardiomyocyte apoptosis via the PI3K/AKT/mTOR pathway. Evid Based Complement Alternat Med 2017: 2590676, 2017.

32. Yang J, Pi C and Wang G: Inhibition of PI3K/Akt/mTOR pathway by apigenin induces apoptosis and autophagy in hepatocellular carcinoma cells. Biomed Pharmacother 103: 699-707, 2018.

33. Polivka J Jr and Janku F: Molecular targets for cancer therapy in the PI3K/AKT/mTOR pathway. Pharmacol Ther 142: 164-175, 2014.

This work is licensed under a Creative Commons Attribution-NonCommercial-NoDerivatives 4.0 International (CC BY-NC-ND 4.0) License. 